Z. klin. Chem. u. klin. Biochem.

7. Jg., S. 501-504, September 1969

\title{
Die Photosonde
}

\author{
Ein Versuch, mittels Faseroptiken die photometrische Messung zu vereinfachen \\ Von H. KeLLER \\ Klinisch-Chemisches Institut, Katbarinenhospital Stuttgart
}

(Eingegangen am 19. Mai 1969)

\section{Herrn Prof. Dr. Hans Netter, Kiel, in Dankbarkeit und Verebrung zur Vollendung des 70: Lebensjabres gevidmet}

\begin{abstract}
Nach einer kurzen Ưbersicht über den Stand der Technik von Faseroptiken wird die Verwendung einer Faseroptik zur photometrischen Messung mit dem Photometer Eppendorf beschrieben.

Der Vorteil dieser Anordnung besteht darin, die Meßgeschwindigkeit zu erhöhen, ohne daß die Präzision abnimmt. Der versuchsweise Aufbau ist auch geeignet, Reflexionsmessungen z. B. an Dünnschicht-Chromatographie-Platten durchzuführen. Weitere Einsatzmöglichkeiten für Faseroptiken im klinisch-chemischen Laboratorium werden kurz diskutiert.
\end{abstract}

\section{The photo-probe. An attempt to simplify photometric measurement by the use of fibre optics}

Following a short review of the present techniques of fibre optics, the use of fibre optics for photometric measurements with the Eppendorf photometer is described.

The advantage of this arrangement is an increased rate of measurement without loss of precision. The experimental arrangement is also suitable for reflection measurements, e. g., on thin layer plates. Further possibilities for the application of fibre optics in the clinical chemical laboratory are briefly discussed.

Lichtleitfasern bestehen aus einer hochbrechenden Glasfaser, die mit einem nichtbrechenden Glasmantel umschmolzen ist. Dadurch entsteht eine praktisch verlustlose Totalreflexion an den Grenzflächen. Da die Grenzschicht ins Innere der Faser verlegt ist, wird sie gegen äußere Einflüsse unempfindlich. Andererseits ist für die (weitgehend) verlustlose Reflexion eine exakte Grenzfläche zwischen Kern- und Mantelglas erforderlich. Sie darf keinerlei Inhomogenitäten enthalten. Die Manteldicke der heute industriell gefertigten Lichtleitfasern beträgt etwa $2 \mu \mathrm{m}$, die Dicke des Kernglases schwankt zwischen $5 \mu \mathrm{m}$ und einigen Millimetern. Übersteigt der Durchmesser des Kernglases eine gewisse Grenze, so spricht man nicht mehr von Fasern, sondern von Lichtleitstäben. Dünne Glasfasern sind biẹgam genug, um daraus flexible Lichtleiter herzustellen. Damit die Bedingungen für die Totalreflexion weiter bestehen, darf der Biegeradius das 20 fache des Faserdurchmessers nicht unterschreiten. Die industriell gefertigten Fasern haben heute meist einen Durchmesser von etwa $70 \mu \mathrm{m}$, wodurch sehr enge Radien erreicht werden können. Andererseits reicht diese Dicke noch aus, um Rasterbilder zu übertragen, die für viele Fragestellungen eine hinreichend kleine Rasterung besitzen (Ưbersicht bei 1 ).

Die Durchlässigkeitskurven flexibler Lichtleiter wurden in den letzten Jahren laufend verbessert. Der Lichtverlust setzt sich aus drei Anteilen zusammen:

1. Dem sogenannten Grundverlust, der durch die Reflexionsverluste am Anfang und Ende des Lichtleiters an den Endflächen entsteht.

2. Dem „Ballastquerschnitt“, der dadurch zustandekommt, daß nicht der gesamte beleuchtete Querschnitt zur Lichtleitung herangezogen werden kann, da die Hohlräume zwischen den Fasern die wirksame Fläche einschränken.

3. Einem Verlust an Lichtenergie in Abhängigkeit von der Faserlänge. Dabei handelt es sich um Verluste durch Absorption, gestörte Totalreflexion und gebrochene $\mathrm{Fa}$ sern.

Handelsübliche flexible Lichtleiter können nicht zur Bildübertragung eingesetzt werden, da die Faserordnung dazu nicht ausreicht. Im Gegensatz dazu sind die flexiblen Bildübertragungskabel aus dünnsten Lichtleitfasern $(5-20 \mu \mathrm{m})$ zusammengesetzt, die am Anfang und Ende genau geordnet sind.

Dort, wo es nur darauf ankommt, Lichtenergie zu übertragen, kann auf diese Ordnung verzichtet werden und man kann von den sehr viel einfacher herstellbaren (und damit viel billigeren), ungeordneten Lichtleitfasern $\mathrm{Ge}-$ brauch mähen.

Bei der Photometrie in jeder Form spielt die Küvette, die das Meßgut aufnimmt, eine fundamentale Rolle. An ihre Präzision hinsichtlich des Wandabstandes, der Glasqualität und der Halterung im Strahlengang auf der optischen Bank, müssen höchste Ansprüche gestellt werden, wenn zuverlässige Resultate erhalten werden sollen. Die Küvette befindet sich bei den meisten Photometern im Inneren des Meßinstrumentes häufig in einem abgedunkelten, schwer zugänglichen Lichtschacht. Füllung und Entleerung der Küvetten müssen jedoch besonders sorgfältig durchgeführt werden, damit die Verschleppungsfehler klein bleiben. Die Arbeitsgänge sind daher in der Regel sehr viel zeitaufwendiger als der eigentliche Meßvorgang. Schließlich ist die Küvette bestimmend für das benötigte Mindestvolumen des Meßgutes 
Es ist überraschend, daß in den 30 Jahren, seit das Photometer zum integralen Instrument analytischer Laboratorien geworden ist, so gut wie nichts an den Küvetten geändert wurde. Eine Prüfung, ob Faseroptiken in der Photometrie eingesetzt werden können, um mit ihrer Hilfe neue, vereinfachte und verbesserte Meßtechniken zu erreichen, liegt nahe.

Grundsätzlich wird davon ausgegangen, das einfallende Licht mittels einer Faseroptik aus dem Instrument herauszuführen, es der Probe zuzuleiten und nach Absorption oder Reflexion in einem zweiten Faserstrang zum Empfänger wieder zurückzuführen (Abb. 1). An welcher
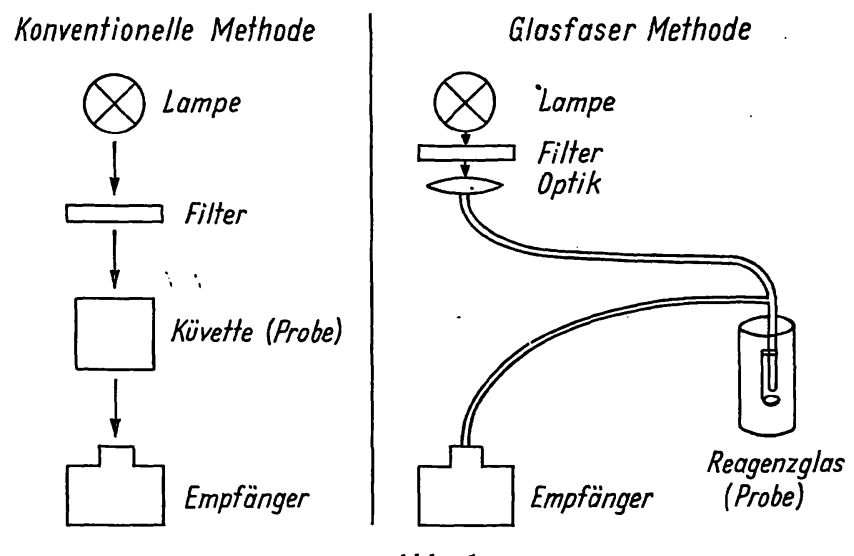

Die konventionelle Photometrie und der Einsatz von Glasfaseroptiken sind schematisch gegenübergestellt

Stelle dabei die Monochromasierung erfolgt, ist theoretisch zunächst gleichgültig. Soll die Absorption gemessen werden, so kann man sich eines zweiarmigen flexiblen Lichtleiters bedienen und den einen Arm zur Lichtzufuhr und den anderen zur Lichtrückleitung benutzen und die vereinigten Faserbündel in die Probe tauchen. Das aus dem Faserteil A ausfallende Licht (Abb. 2a) durchdringt die Meßlösung, trifft auf eine reflektierende Schicht - z. B. einen Spiegel - und die reflektierten Strahlen werden vom Faserteil B wieder aufgenommen. Ist eine Schichtdicke von $1 \mathrm{~cm}$ erwünscht, so muß der Abstand zwischen Faserendfläche und Reflexfläche $0,5 \mathrm{~cm}$ betragen. $\mathrm{Zu}$ beachten ist, daß naturgemäß nur ein Teil des reflektierten Lichtes über B zum Empfänger gelangt, ein zweiter Teil wird über $\mathrm{A}$ wieder zur Lichtquelle zurückgeleitet.
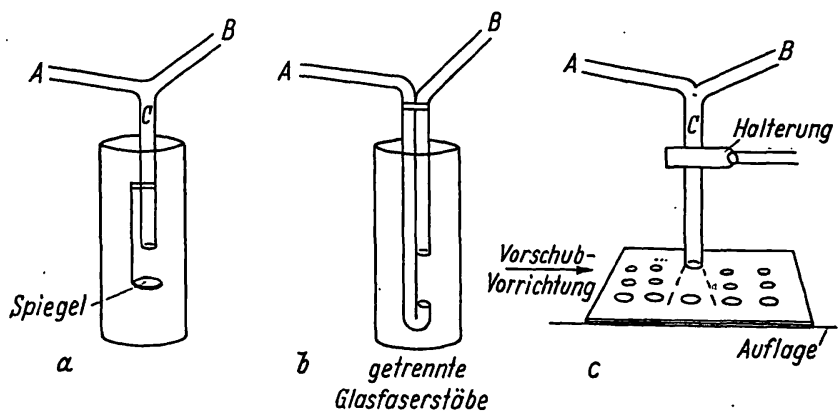

Abb. 2

Photometrie mittels Glasfaseroptik

a) Absorptionsmessung in Lösungen gegen einen Spiegel, b) Absorptionsmessung über Glasfaserstäbe, c) Reflexionsmessung an einer Dünnschicht-Chromatographie-Platte
Eine andere Möglichkeit besteht darin, zwei getrennte Faserstäbe zu benutzen, wie es in der Abbildung $2 \mathrm{~b}$ dargestellt ist.

$\mathrm{Da}$ die Faserstäbe sehr eng gebogen werden können, ist eine solche Anordnung technisch ohne weiteres möglich und wird zweifellos auch zu brauchbaren Resultaten führen. In diesem Fall muß der Abstand allerdings $10 \mathrm{~mm}$ betragen (wenn der Lichtweg in dieser Länge gewünscht wird). Det Vorteil dieses Systems dürfte in der übersichtlichen Anordnung bestehen.

Soll in Reflexion gemessen werden, so genügt es, den Lichtleiter in geeignetem Abstand über dem zu messenden Objekt zu fixieren. Auf diese Weise können unschwer Anordnungen zusammengestellt werden, die zur Ausmessung von Dünnschichtchromatogrammen geeignet sind (Abb. 2c).

Für photometrische Zwiecke ist es wichtig, den Energieverlust durch Streustrahlung so klein wie möglich zu halten. Die Anordnung der Lichtleitfasern dürfte daher theoretisch dann optimal sein, wenn die Energiezufuhr als Kern, während die Energierückleitung als Mantel ausgebildet ist.. Diese Anordnung ist im Augenblick jedoch noch nicht erhältlich.

Die Versuche, über die hier erstmalig berichtet wird, wurden mit einem handelsüblichen zweiarmigen, flexiblen Lichtleiter der Firma Jeaner Glaswerke Schott \& Gen., Mainz, durchgeführt. Jeder der beiden Arme ist rund und hat einen Durchmesser von $4 \mathrm{~mm}$, die vereinigten Arme haben einen Durchmesser von $5,8 \mathrm{~mm}$. Die beiden Arme sind mit PVC ummantelt. Die Vereinigungsstelle ist bis ans Ende in einer Messingkopfhülse zusammengefaßt.

Zur Absorptionsmessung wurde über den unteren Teil eine PVC-Kappe geschoben, die in ihrem Inneren einen Spiegel enthält (Abb. 3). Entsprechende Kunststoffhalte-

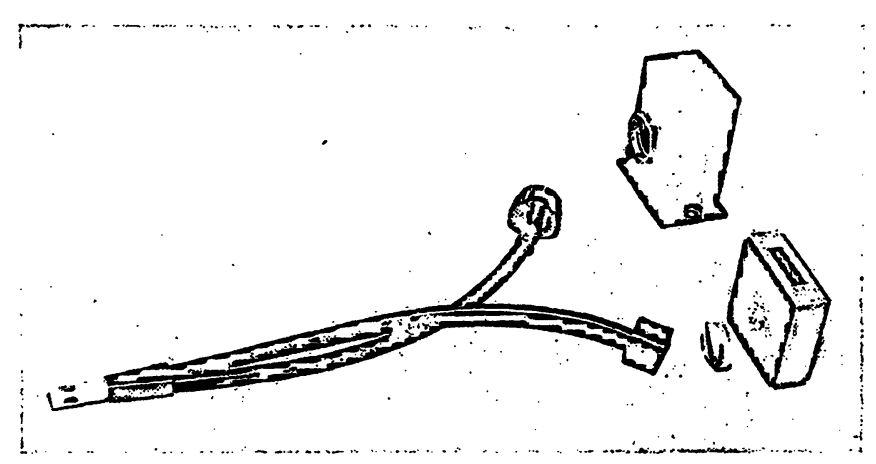

Abb. 3

Faseroptik mit PVC-Kappe und den Anschlußstüçken für das SEVGehäuse, für die Linse und das Filter. Am Boden der PVC-Kappe befindet sich ein Spiegel

rungen sorgen für eine Fixierung der beiden Arme des Lichtleiters einerseits am Filter, andererseits am Gehäuse des Sekundär-Elektronen-Vervielfạchers des Photometers Eppendorf.

Da das quasi parallele Quecksilberlicht des Photometers nach dem Durchtreten des Filters einen Durchmesser von etwa $21 \mathrm{~mm}$ hat, der des Lichtleiters aber nur $4 \mathrm{~mm}$ beträgt, gehen bei dieser Anordnung etwa 
$85 \%$ Lichtenergie verloren. Durch entsprechende Verstärkung kann der Verlust zwar kompensiert werden, doch gilt dies nur für die relativ starken Quecksilberbanden, wie z. B. $546 \mathrm{~nm}$ oder $578 \mathrm{~nm}$. Die schwächeren Banden können nicht mehr, oder nur noch mit maximaler Verstärkung kompensiert werden. Es hat sich deshalb als zweckmäßig erwiesen, diesen Lichtverlust durch Einschalten einer Sammellinse kurzer Brennweite auszugleichen. Hier wurde dazu eine bikonvexe Glaslinse mit einer Brennweite von $25 \mathrm{~mm}$ verwendet. Ihr Durchmesser betrug $21 \mathrm{~mm}$. Sie wurde auf die der Lichtquelle zugekehrten Seite des Filters aufgeklebt und auf diese Weise eine Verstärkung um den Faktor 10 der Lichtausbeute erreicht (Abb. 4 u. 5).

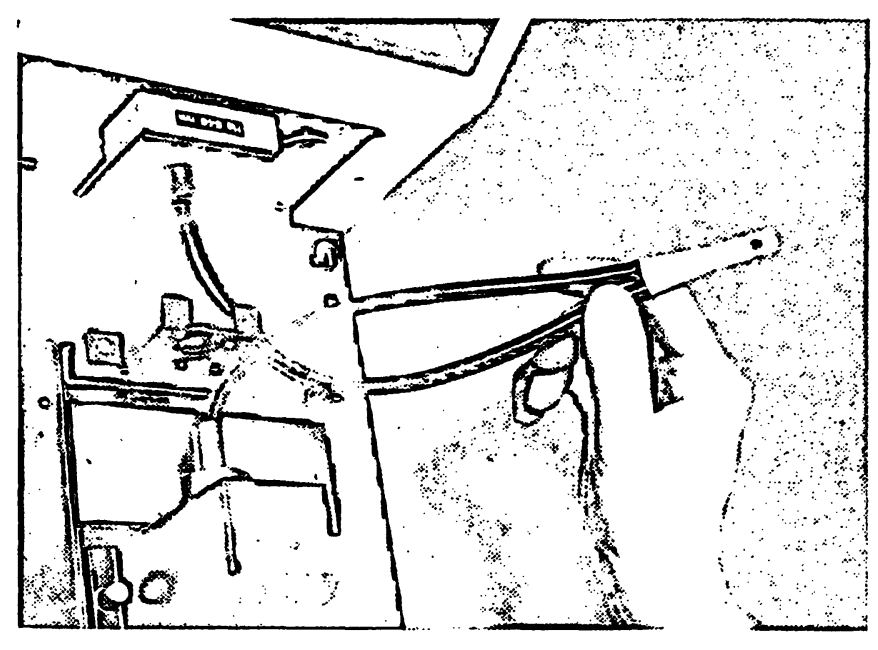

Abb. 4

Faseroptik im Photometer Eppendorf meßbereit montiert

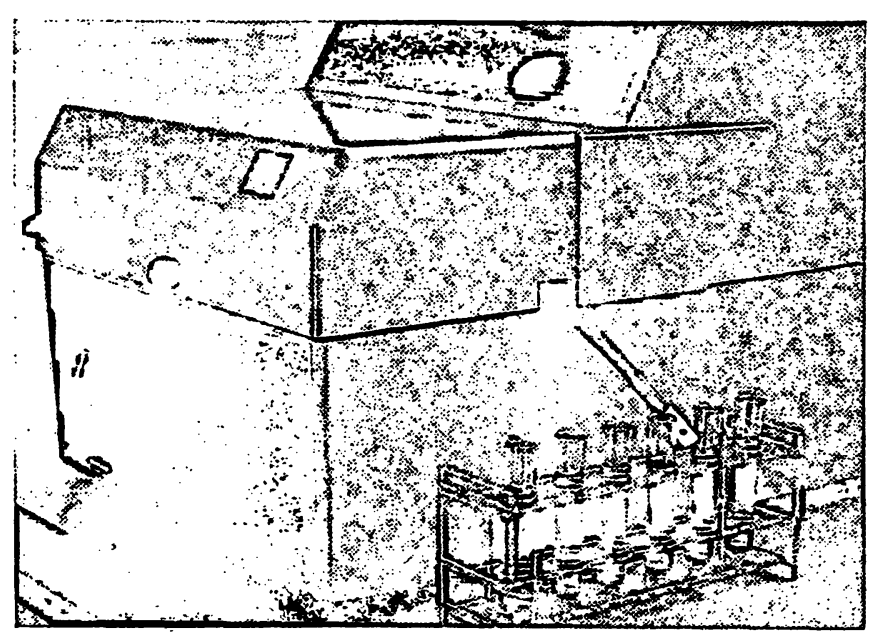

Abb. 5

Die meßbereite Photosonde

Der Meßkopf leuchtet durch Streustrahlung

Die Erprobung der Anordnung lieferte schon beim ersten Versuch ein überraschend gutes Ergebnis. Die wiedergegebene Biuret-Eichkurve (Abb. G) ist fast identisch mit der Eichkurve, die mit einer Präzisionsküvette mit den gleichen Farblösungen gewonnen wurde. Die geringgradig größere Steilheit der Meßsondenkurve ist zweifellos darauf zurückzuführen, daß die Distanz zwischen Faserende und Spiegel nicht exakt 5,0 $\mathrm{mm}$ sondern

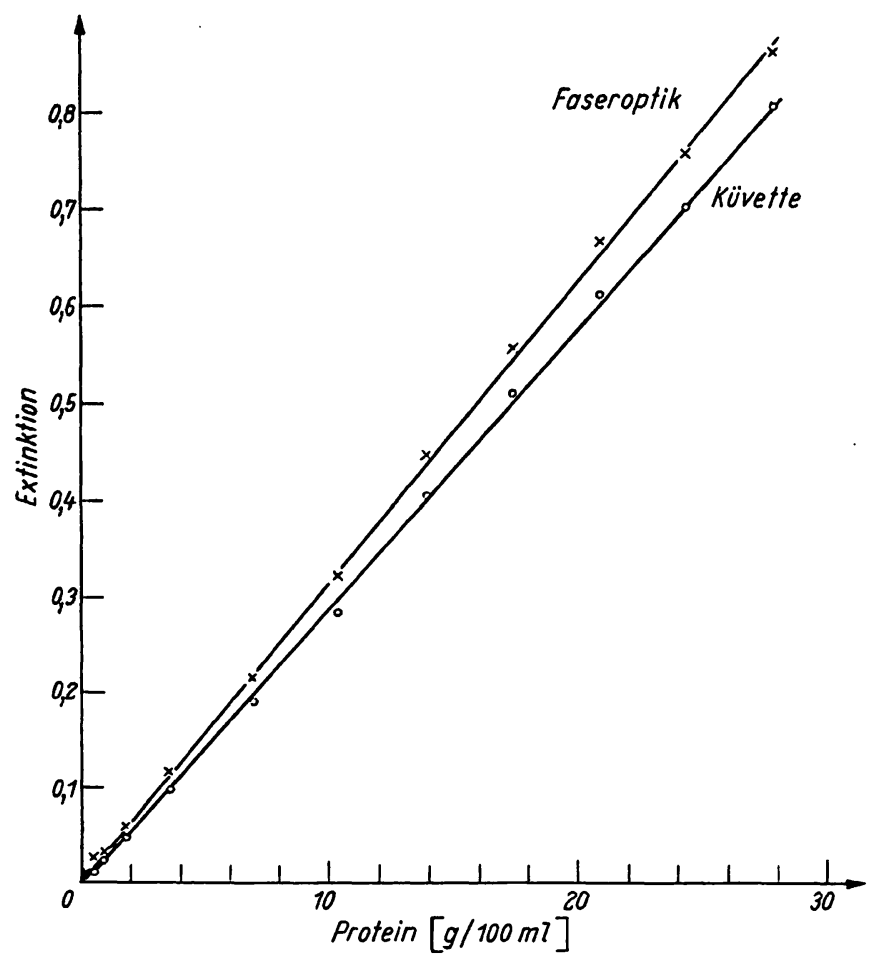

Abb. 6

Biuret-Eichkurve

etwas mehr beträgt. (Wahrscheinlich wurde die Dicke der Klebschicht für den Spiegel überschätzt.)

Frappierend ist, mit welcher Geschwindigkeit gemessen werden kann. Die Meßsonde wird von einem Glas in das nächste Glas getaucht; geschwindigkeitsbestimmend ist lediglich die Zeigereinstellung und Ablesung oder das Ausdrucken über einen Analog-Digital-Wandler. Der Verschleppungsfehler scheint nach den vorliegenden Erfahrungen trotz der relativ ungünstigen Anordnung minimal zu sein. (Wenn demnächst eine komfortablere Ausführung zur Verfügung steht, werden auch Messungen in sehr kleinen Gefäßen - z. B. im unteren Drittel eines Eppendorf-Gefäßes - ohne nennenswerten Verschleppungsfehler möglich sein.)

Zur Zeit wird für uns ein Faseroptik-System zusammengestellt, das aus Quarzteilen besteht, um auch im UVBereich bis zu $200 \mathrm{~nm}$ messen zu können. Die beiden Lichtleiter werden voraussichtlich eine Länge von etwa $50 \mathrm{~cm}$ haben und sich in einem massiven ummantelten Quarzstab mit großem Öffnungswinkel vereinigen. Auf diese Weise soll die Lichtausbeute noch weiter verbessert und zugleich die Handhabung der Anlage erleichtert werden. Weiter ist daran gedacht, auf diese Weise auch die Möglichkeit zu schaffen, die Photosonde für automatische Meßplätze heranzuziehen. Besonders im Rahmen der Automation, Mechanisierung und Teilmechanisierung analytischer Laboratorien, dürften die Vorteile der Meßsonde augenfällig sein.

Lichtleiter und Faseroptiken haben heute bereits einen vielfältigen und keineswegs auf die medizinische Endoskopie beschränkten Anwendungsbereich. Sie finden in der Technik als Bildwandler, als Lichtschranken, seit einiger Zeit sogar in der Waffentechnik vielfältige Ver- 
wendung. Von besonderem technischem und wirtschaftlichem Interesse wird ihre breitere Nutzung in der Nachrichtentechnik sein. Auch für die Fernsehtechnik wird von Faseroptik, insbesondere von sogenannten Faserplatten, noch einiges erwartet (2).

Auf dem Gebiet der analytischen Chemie sind Faseroptiken bisher nur spärlich angewandt worden: Orientierende Versuche ohne quantitative Auswertung haben Hamman und Martin (4) mitgeteilt. Beroza und Mitarbeiter (3) bedienten sich einer Faseroptik zur Auswertung von Dünnschicht-Chromatogrammen, von der Firma Fischer-Scientific ist ein automatischer Titrator be- schrieben, in dem zwei Glasfaserstäbe in die Titrierlösung eintauchen und den Titriervorgang steuern (Literatur bei 2). Die hier vorgestellte Lösung stellt einen neuen Versuch dar, dessen gutes Gelingen weitere Entwicklungsarbeiten in dieser Richtung rechtfertigen.

Der Verfasser hat Herrn Dipl.-Phys. A. JACOBSEN, Jenaer Glas- werke Schott \& Gen., Mainz, für die großzügige Unterstützung dieser Arbeit sehŕ zu danken. Ebenso ișt er für vorzügliche technische Assistenz Frau Eva Neumans und Fräulein Verena Wolf zu Dank verpflichtet. Die Anschlußstücke mit Linse und Spiegel wurden vom Technischen Büro Jörg LIMBERg, Stuttgart-Feüerbach, konstruiert.

\section{Literatur}

1. JACOBSEN, A. und W. Rimkus, Feinwerktechnik 71, 111 (1967). 2. Crum, J. K., Analytic. Chem. 41, 26 A (1969). - 3. BerozA, M., K. R. Hill und K. H. Norris, Analytic. Chem. 40, 1608
(1968). - 4. Hamman, B. L. und M. M. Martin, Analytic. Biochem. 15, 305 (1966).
Prof. Dr.H. Keller 7 Stuttgart 1

Kriegsbergstraße 60 


\section{Unsere Gefriertrocknungsanlage GT 2 ist so vielseitig, weil sie Bauteile fidur jeden Einsatzbereich hat.}

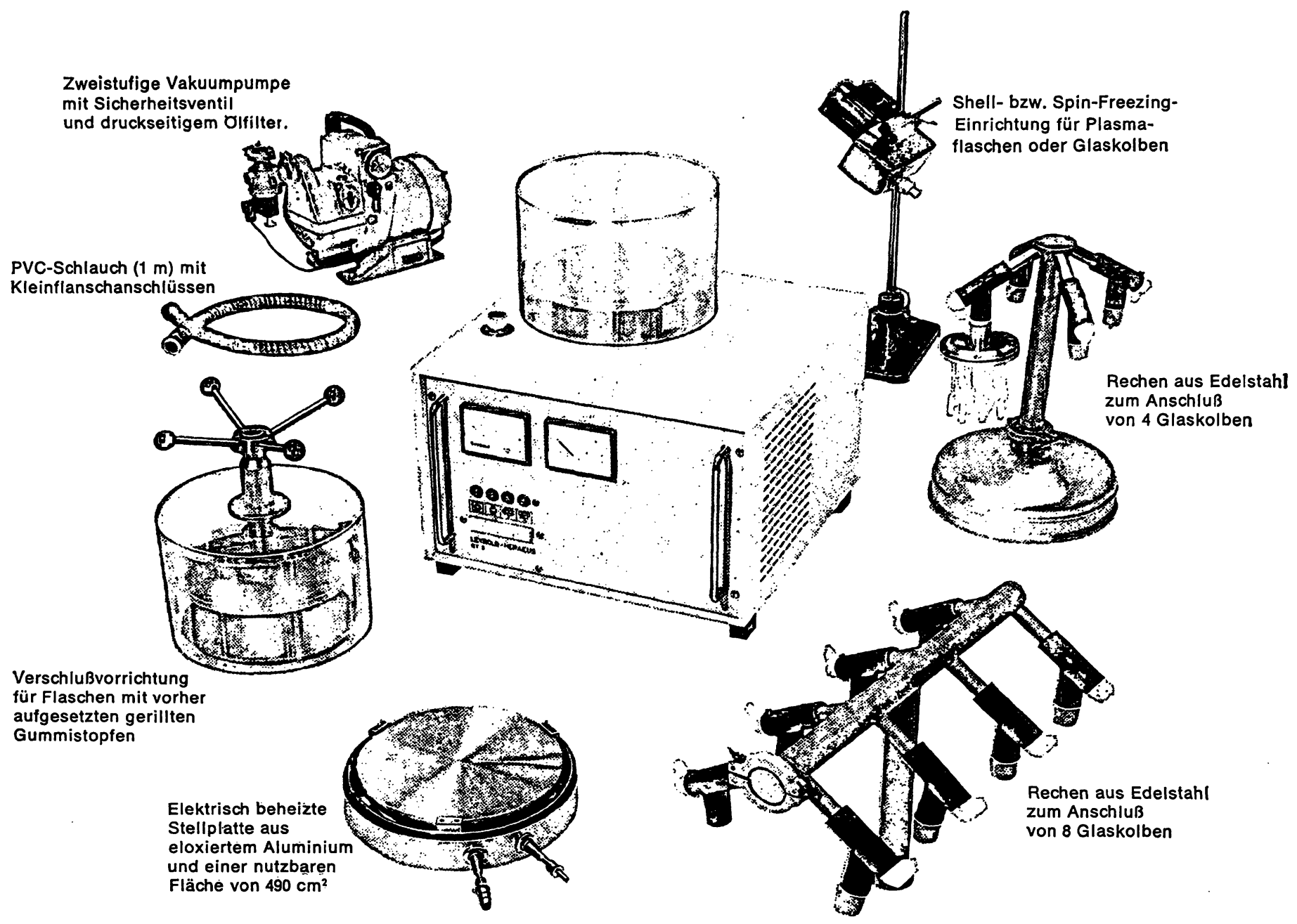

Ganz gleich was Sie gefriertrocknen wollen, ob Pharmazeutika, Bakterien, Gewebe, Seren, Pflanzen, Erdproben, Kleinlebewesen, Insektén oder Lebensmittel - die GT 2 ist einfach universell. Alles bleibt natürlich in Aussehen, Struktur und Zusammensetzung.

Verfahrenstechnische Vorteile :

(2) Einfache Bedienung

- Eingebaute Sicherheitsventile

- Wesentliche Zeitersparnis (LH-Patent)
Vorrichtung zum Messen derTemperatur des Eises im Produkt. Sie können das Ende der Trocknung feststellen, den Druck regulieren, den Wärmeübergang verbessern und damit den Trocknungsprozeß beschleunigen.

Geringer Platzbedarf

Es liegt also ganz bei Ihnen, ob Sie zukünftig erfolgreicher arbeiten wollen.

Ubbrigens: wenn Sie es wünschen, führen wir für Sie Versuche auch in unseren Labors durch.

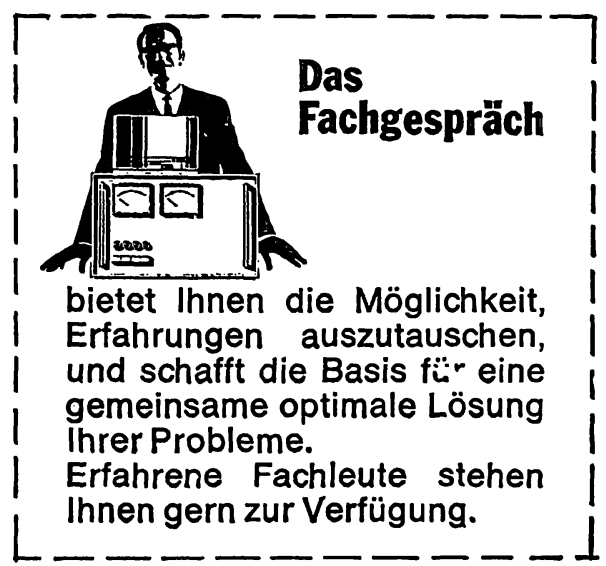




\section{Glucose-6-phosphat Dehydrogenase}

Sigma stellt jetzt als erste Firma kristalline Glucose-6-phosphat-Dehydrogenase sowohl aus Backer- als auch aus Torula-Hefe her. Bisher wurde Glucose-6-phosphat-Dehydrogenase aur aus Bravere-Hefe hergestell (Noliman und Milarbeiter, J. biol. Chemistry 236, 1225 (1961). Eine vergleichende Unersuchung dieser drei Enzyme kistein. hoher lonenstärke in das Apoenzym, wobei sie ihr gebundenes NADP abgeben (Yue und Mitarbeiter, Biochemistry, USA, 6, 1174 [1967]). Das Enzym aus Torula ist bei Raumtemperafur sowohl als wäßrige Suspension als auch als Lyophilisat völlig stabil. Unseres Wissens ist das bisher noch nirgendwo erreicht worden. Sigma bietet jetzt die kompletteste Liste von Alucose-6-phosphat-Dehydrogenase einschließ lich höchster Reinheiten und Spezifitäten an.

Vorläufige Beobachfungen am Bäcker- bzw. Torula-Hefe-Enzym zeigen:

1. Das Torula-Enzym ist erstaunlich stabil

2. Das Torula-Enzym benötigł ełwa $2 \times$ mehr Glucose-6-phosphat für maximale Aktivitäł

3. Beide Enzyme unterliegen der "Sulfat-lonen"-Hemmung, jedoch in unterschiedlichem Maße

4. Die Hemmung des Bäcker-Hefe-Enzyms kann durch Glucose-6-phosphat-Konzentrationen $>3$ mM überwunden werden.

\begin{tabular}{|c|c|c|c|}
\hline $\begin{array}{c}\text { Sulfat- } \\
\text { Konzentration }\end{array}$ & Torula G-6-PDH & $\begin{array}{l}\text { Effekt } \\
\text { Bäcker-Hefe-G-6-PDH }\end{array}$ & $\begin{array}{l}\text { Glucose-6-phosphät- } \\
\text { Konzentration }\end{array}$ \\
\hline $\begin{array}{l}0,001 M \\
0,01 M \\
0,1 M\end{array}$ & $\begin{array}{l}20 \% \text { Hemmung } \\
80 \% \text { Hemmung } \\
99 \% \text { Hemmung }\end{array}$ & $\begin{array}{l}66 \% \text { Hemmung } \\
50 \% \text { Hemmung } \\
93 \% \text { Hemmung }\end{array}$ & $\begin{array}{l}1 \times 10^{-4} M \\
1 \times 10^{-4} M \\
1 \times 10^{-4} M\end{array}$ \\
\hline
\end{tabular}

Um die Dissoziation in das Apoenzym bei der hohen lonenstärke von $70 \%$ gesättigter Ammoniumsulfatlösung ziu verhindern, haben wir unseren Kristallsuspensionen (Typ VII und XI) drei Mole weiteres NADP pro Mol G-6-PDH hinzugesetzi. Wir bieten auch sulfatfreie Präparate (Typ XII und XV) beider Hefestämme an. Das sind Lyophilisate (15\% Salz und $85 \%$ Eiweiß), die G-6-PDH teils als Apoenzym, teils als NADP-Enzym enthalten. Sie enthalten kein zugesetztes NADP und sind deshalb sehr geeignet für Untersuchungen mit der Technik des "Enzymatic Cycling“.

Wenn hohe Stabilität erforderlich ist und nur eine geringe Sulfat-Konzentration erreicht werden darf, verwendet man das TorulaHefe-Enzym. Wenn höhere Sulfat- und sehr geringe Glucose-6-phosphat-Konzentrationen vorliegen, wird das Bäcker-Hefe-Enzym benutzt.

Definition der Enzymeinheit: Eine Einheit oxydiert $1 \mu \mathrm{Mol}$ Glucose-6-phosphat pro Minute in Gegenwart von NADP bei pH 7,4 und $25^{\circ} \mathrm{C}$.

Produkt

$\mathrm{Nr}$

Typ V: Aus Bäcker-Hefe

Unser alter Ammoniumsulfat-Trockenkuchen, unterliegt Sulfat-Hemmung (s. o.).

Aktivität: > 150 Einheiten/mg Protein. Hexokinase und 6-Phosphogluconat-Dehydrogenase höchsłens in Spuren enthalten.

Typ VII: Aus Bäcker-Hef

G 7877 Kristallsuspension in 3,2 M Ammonium sulfat.

Aktivität: $300-400$ Einheiten/mg Pro-

tein. Sulfat-Hemmung (vgl. o.)
150 Einheiten $\$ 9,50$ 250 Einheiten 12,50 500 Einheiten 21,25 1000 Einheiten 35,50 2000 Einheiten 57,75 5000 Einheiten 147,50

Typ XV: Aus Bäcker-Hefe

Aktivität vorher-

G 6378 Kristallisiert, lyophilisiert, sulfatfre Aktivität: Etwa 200-400 Einheiten/mg Protein. Enthält etwa $15 \%$ Magnesium acetat. Das ist wahrscheinlich das erste Angebot kristalliner G-6-PDH aus Bäcker-Hefe.

Wird mit reinem Wasser oder Puffer aufgefüllt.
Anm, Lyophilisa

in Ampullen

100 Einheiten $\$ 6,25$

500 Einheiten 21,25

1000 Einheiten 35,50

2000 Einheiten 57,75

5000 Einheiten 147,50
250 Einheiten 12,50
100 Einheiten $\$ 5,00$ 250 Einheiten 10,00 500 Einheiten 17,00 1000 Einheiten 28,50
2000 Einheiten 46,25

Produkt

Nr.

\section{Typ XI: Aus Torula-Hefe}

G 8878 Kristallsuspension in 2,6 M Ammoniumsulfat.

Aktivität: Etwa 300-400 Einheiten/mg Protein. Sehr geringe oder keine Beimengung folgender Enzyme: 6-Phosphogluconat-Dehydrogenase, Hexokinase, dase.

Völlig stabil bei Raumtemperafur. Sulfat-Hemmung (s. o.).

Dieses Präparat ist ökonomischer als das vergleichbare aus BäckerHefe, ausgenommen für Systeme mit niedriger Substratkonzentration.

Typ X11: Aus Torula-Hefe

Kristallisiert, lyophilisiert

Aktivität: $200-300$ Einheiten/mg

Protein. Sulfatfrei.

Augenblicklich enthalten die Chargen

etwa 5\% Magnesiumacetat und $10 \%$

Ammoniumacetat. (Es ist vorgesehen,

dieses Präparat in näherer Zukunft

ammoniumfrei zu machen.)

Völlig stabil bei Raumtemperatur, auch

nach Auffüllung mit Wasser oder Puffer, wenn bakterienfrei.

Reinheitsgrad III: Rohpulver, aus

G 1878 Bäcker-Hefe Aktivität: Etwa 0,3-1,0 Einheiten/mg. $\quad 300$ Einheiten $\quad 30,00$ Enthält gewöhnlich Hexokinase und 5000 Einheiten 300,00 6-Phosphogluconat-Dehydrogenase.

Versand in alle Welt per Luftpost, Fracht und Versicherung frei.

Sigma-Reagenzien sind in der ganzen Welt durch den Fachhandel oder direkt aus St. Louis beziehbar.

Telegramme: SIGMACHEM, St. Lovis, Missouri

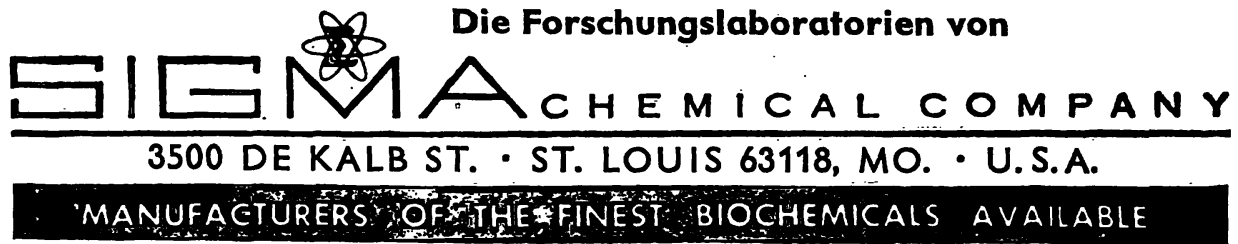

Vertretung in England:

SIGMA LONDON Chem. Co. Ltd. - 12, Lettice St., London S. W. 6 ENG. 